\title{
An Unusual Presentation of Coronary Artery Fistula in Athlete - Case Report
}

\author{
Ale xander S. Kisko ${ }^{1, *}$, Lubica De rnarova ${ }^{1}$, Jan Kmec ${ }^{1}$, Marika Vere ${ }^{2}$, Anna Hudakova ${ }^{1}$, \\ Marta Jakubikova ${ }^{1}$, Nelli Kis hko ${ }^{3}$ \\ ${ }^{1}$ Faculty of Health Care, Presov University in Presov, Presov, 080 01, Slovakia \\ ${ }^{2}$ Nuclear Medicine, Sekcov Polyclinic, Presov, 08001 Slovakia \\ ${ }^{3}$ Medical Faculty, Uzhgorod National University, Uzhgorod, 080 01, Ukraine
}

\begin{abstract}
A 32-year-old active cyclist was referred for the evaluation because of syncope he had performed in preseason. He was found to be normostenic, acyanotic, normotensive with clear lungs and a regular pulse of $60 \mathrm{bpm}$, with normal dual heart sounds and a grade Levine $2 / 6$ continuous diastolic murmur in the second intercostal space of the left parasternal area. He had no family history of premature card iac death and his lipids were normal. Rest ECG showed a regular sinus rhythm of $62 \mathrm{bpm}$ with incomplete right bundle branch block and no significant ST-T changes. A transthoracic ECHO in parasternal short-axis view revealed an anomalous colour flow jet in diastole arising fro $m$ the lateral wall into the main pulmonary artery and coronary artery fistula with non-significant left-to-right shunt $(\mathrm{Qp} / \mathrm{Qs}$ ratio 1.2).ca me under suspicion. In contrast, it has not been confirmed clearly by the transoesophageal ECHO. Coronary angiography was without coronary stenosis and confirmed a 'serpentine' anomalous drainage supposedly from left anterior descending artery to the main pulmonary artery. A complex anato my of sacculary dilated fistula that originates from the proximal left anterior descending artery and drainages the main pulmonary artery was showed in detail by a 64 slice MDCT scanning. Myocardial Tc $-99 \mathrm{~m}$ Myoview perfusion SPECT imaging showed no perfusion defects in maximal physical stress and follow-up without intervention was suggested. In addition, due to the results of holter ECG monitoring, head-up tilt testing, carotid sinus massage and programmed atrial stimulation syncope was concluded as vasovagal, but its nature still remains discussible. After a three year follow-up patient remains asymptomatic and recently performed control myocardial perfusion SPECT showed no signs of stress related myocardial ischemia. In conclusion, several imaging techniques are needed for an accurate diagnosis of coronary fistula and for the suggestion of proper further management. In some cases syncope may be the first man ifestation of CAF, but it is still unclear if it is directly related to the coronary anomaly.
\end{abstract}

Keywords Coronary Artery Fistula, Syncope, Doppler Colour Flow Imaging, MDCT Scanning, Tc-99m Myoview Perfusion SPECT

\section{Introduction}

Congenital coronary artery fistula (CAF), first described by Krause in 1865 is characterized by normal aort ic orig in of the coronary artery but with a fistulous communication with the great vessels as well as all cardiac chambers. CAFs are rare, predomin antly congenital or occasionally acquired (such as stab or projectile injuries, post-coronary angioplasty) coronary artery anomalies that can orig inate fro $m$ any of the three major coronary arteries. The evolution of the coronary artery network involves complex embryology. In early foetal development, persistence of myocardial sinusoids and the subsequent connection with the

* Corresponding author:

alexander.kisko@unipo.sk(Alexander S. Kisko)

Published online at http://journal.sapub.org/cmd

Copyright (C) 2012 Scientific \& Academic Publishing. All Rights Reserved endothelial buds that originated from the base of truncus arteriosus forms the basis for abnormal coronary fistulas.

Although coronary fistula is rare, it is one of the most common among the coronary artery anomalies. CAF most commonly affects the right side of the heart. It may occur isolated or along with congenital heart diseases[1]. According to the global experience the current characteristics of congenital coronary fistulas in adults had changed in the last decade. It was reported in 2006 that the origin of the fistulas was from the left coronary artery in $58 \%$ and from the right in $42 \%$ of patients and in the recently published review the overall figures were $69 \%$ and $31 \%$, respectively. In 2006, the fistulas were unilateral, bilateral and multilateral in $93 \%, 6 \%$ and $1 \%$, respectively. Currently, the majority, as expected, were unilateral fistulas $(80 \%)$, followed by bilateral fistulas (18\%) and finally multilateral fistulas $(2 \%)$. A mong the unilateral fistulas, the le $\mathrm{ft}$ anterior descending artery was predominantly the origin $(42 \%)$ of the 
fistulas, followed by the right coronary artery $(31 \%)$, the circumflex (20\%) and finally the left main trunk (7\%)[2].

CAFs most commonly drain into the right atriu $m$ or right ventricle, but occasionally can drain into the pulmonary artery. Bilateral coronary fistulas with the coronary artery stenosis are rare[3].

In these coronary artery abnormalities blood is shunted into a great vessel, or other structure, bypassing the myocardial capillary network and may produce steal phenomenon. Unless very large and hemodynamically significant, it is usually asymptomatic in younger patients. With increasing age, symptoms begin to appear, and the incidence of complication rises.

The patients with CAFs are mostly asymptomatic, but some may present with symptoms of fatigue, dyspnoe, angina, arrhythmias, and signs of congestive heart failure, pulmonary hypertension or infective endocarditis. Syncope is very rarely associated with CAF. We report the case of coronary to main pulmonary artery fistula in active athlete first presented with syncope.

\section{Case Presentation}

A 32-year-old active cyclist was referred for the evaluation because of syncope he had performed in preseason. He reported no history of the episodes of shortness of breath or chest pain associated with extreme physical exertion as well as palpitations or other clinical symptoms of cardiac arrhythmias. He was physically absolutely normal with no familial predisposition to sudden cardiac death or ischemic heart disease and normal lipids.

At physical examination patient was found to be normostenic, acyanotic, normotensive, with clear lungs and a regular pulse of $60 \mathrm{bpm}$, with normal dual heart sounds. A grade Levine 2/ 6 continuous murmur could be heard mainly at the level of the second intercostal space of the left parasternal area. There were no signs of heart failure. An electrocardiogram at rest showed a regular sinus rhythm of $62 \mathrm{bpm}$ with incomplete right bundle branch block and no significant ST-T changes.

A transthoracic echocardiography in parasternal short-axis view revealed an ano malous colour flow jet arising fro $m$ the lateral wall into the main pulmonary artery, identifying the fistula drainage site (fig.1) with non-significant left-to-right shunt $(\mathrm{Qp} / \mathrm{Qs}$ ratio 1.2). In contrast, despite various projections, the exact anatomic course of the suspected fistula could not be clearly shown by the transoesophageal echocardiography. Coronary angiography confirmed 'serpentine' fistula with aneurysms corresponding the proximal portion of the left anterior descending artery and the main pulmonary artery with no significant signs of coronary atherosclerosis (fig.2).

The complex anatomy of the fistula was demonstrated in detail by 64 Slice MDCT scanning using multip lanar and 3D reconstruction techniques. It showed a sacculary dilated fistula that originates from the proximal left anterior descending artery and drainages the main pulmonary artery (fig.3).

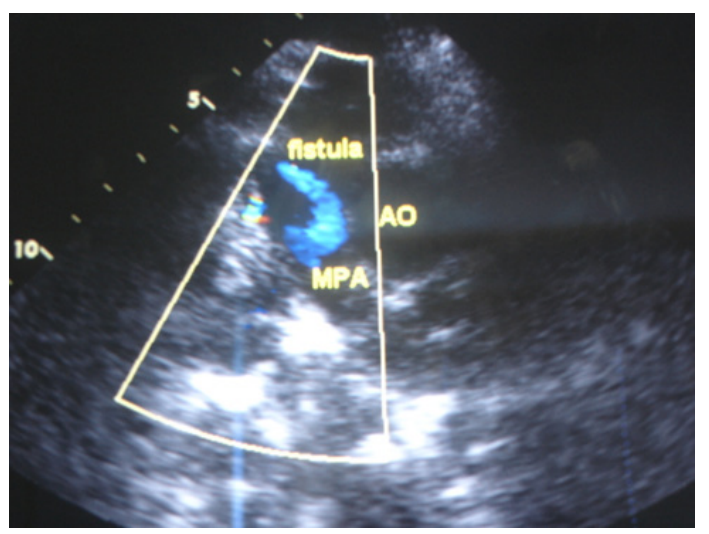

Figure 1. A transthoracic echocardiography in parasternal short-axis view reveals an anomalous colour flow jet at colour Doppler analysis arising from the lateral wall into the main pulmonary artery

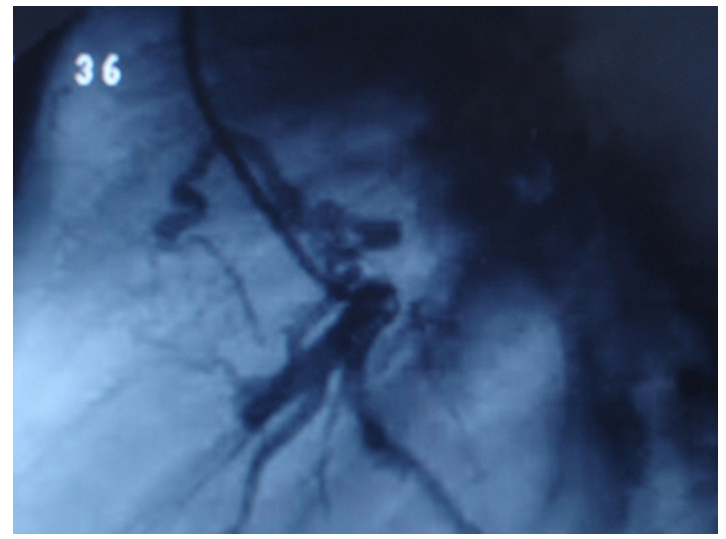

Figure 2. Coronary angiography showing a 'serpentine' fistula with aneurysm corresponding the proximal portion of the left anterior descending artery and the main pulmonary artery

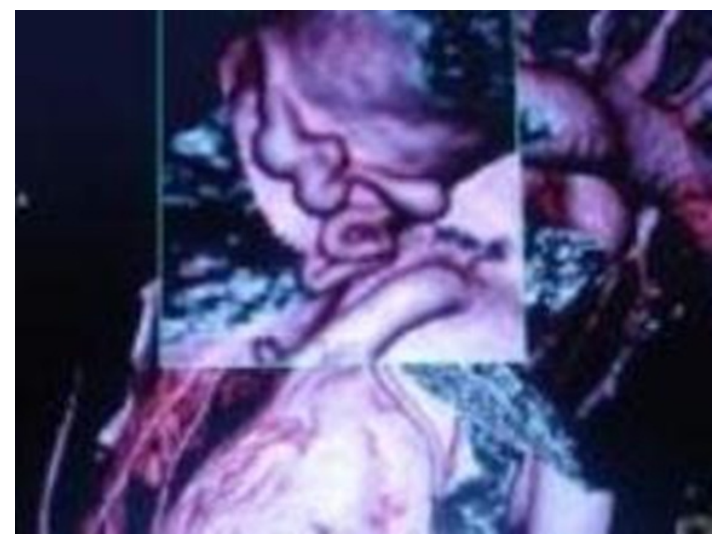

Figure 3. MDCT scanning using multiplanar and 3D reconstruction technique shows a sacculary dilated fist ula that originates from the proximal left anterior descending artery and drainages the main pulmonary artery

To evaluate the hemodynamic significance of detected coronary fistula the stress testing with the bicycle ergo meter was performed in 3-minute steps up to the maximum theoretical heart rate which showed no ST-T change at the heart rate of 181 beats/min (200W, 11,0 MET's) with no symptoms during examination. Stress/Rest Tc-99m Myoview perfusion SPECT scintigraphy revealed no 
perfusion defects and stress-induced myocardial ischemia (fig.4)

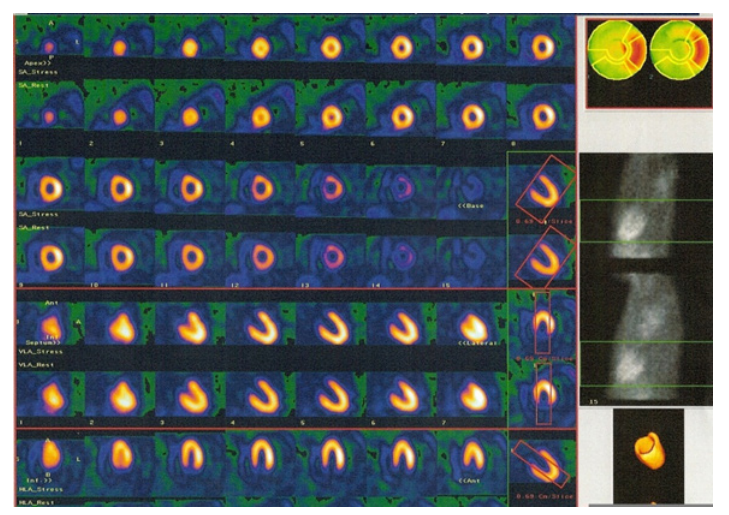

Figure 4. Stress/Rest Tc-99m Myoview perfusion SPECT shows no stress-related perfusion defects

In purpose to evaluate a possible cause of syncope holter ECG monitoring and programmed atrial stimulation were performed with no evidence of the arrhythmogenic nature of syncope episode. According to the results of the patient's response to head-up tilt testing and the carotid sinus massage syncope was concluded as being of vasovagal etiology with no evidences of the dys rhythmic patterns. From these results, the patient was diagnosed as having a hemodynamically non-significant coronary artery to pulmonary artery fistula with no stress-related myocardial is chemia and no evidences of dysrhythmic syncope, but its nature still remains dis cussible.

Conservative follow-up with no intervention procedure was suggested. After a three year follow-up patient remains asymptomatic still participating in cycling tours and other sports events. Recently performed control myocardial Tc-99m Myoview perfusion SPECT scintigraphy showed no evidence of stress-related myocardial is chemia.

\section{Discussion}

The incidence of CAF ranges from $0.2-0.4 \%$ in selected series of congenital heart disease patients, and its incidence in the overall population is estimated to be about $0.002 \%$. The incidence of CAF during 14,708 diagnostic cardiac catheterizations was 0.13 . There was 1 traumatic and 19 congenital cases. All fistulas were small and of little hemodynamic significance. No symptoms or complications could be ascribed to these fistulas. In the large majority no continuous or other murmurs suggested their presence. Surgical treatment for these fistulas was not indicated. The majority of these fistulas arise from the right coronary artery. Left CAF is less common, but usually drains into the right ventricle or right atrium. A combination like the one described in the presented case is unusual since fistulas from the left coronary artery with drainage into the pulmonary artery occurs in only $17 \%[4]$.

The majority of the patients with CAFs are clinically asymptomatic but the long-term outcome is not fully known. The clinical course of CAF may vary greatly from spontaneous closure to severe complications. The patients with coronary fistulas may present with dyspnoea, congestive heart failure, angina, endocarditis, arrhythmias, or myocardial infarction. Syncope is not a typical symptom related to fistulas and is very rare first clinical man ifestation of CAF. It may be associated with some underlying congenital heart defects. Our patient presented with an is olated CAF but no other coexis ting heart abnormalities and it was not fully understood whether a syncope episode was directly related to the fistula. Clinically manifested myocardial ischemia is resulting from fistula steal phenomenon. Coexisting congenital or acquired heart disease is found in $40 \%$ of patients; however, no associated lesion was found to have a specific relationship with CAF.

Up to now there is no generally accepted recommendation about standard diagnostic protocol in case of suspected CAF. Clin ical examination, trans thoracic echocardiography, stress electrocardiogram and stress/rest myocardial perfusion imaging may be first line examinations in evaluation of coronary fistulas[5]. Clinical diagnosis of CAF is difficult due to symptomless or lack of specific symptoms. Clinical manifestations vary considerably and as it was mentioned above the patients with coronary fistulas may present with dyspnea, congestive heart failure, angina, endocarditis, arrhythmias, angina or myocardial infarction, but a majority of patients are asymptomatic as fistulas cause myocardial ischemia only in a small number of them. A continuous murmur heard at the upper sternum border is often presented and is highly suggestive of a coronary fistula Differential diagnosis includes persistent ductus arteriosus, pulmonary arteriovenous fistula, ruptured sinus of Valsalva aneurysm, aortopulmonary window, prolapse of the right aortic cusp with a supracristal ventricular septal defect, internal mammary artery to pulmonary artery fistula, and systemic arteriovenous fistula[6].

Transthoracic echocardiography with colour flow imag ing by an experienced echocardiographer is an ideal routine first-line and in some cases definitive diagnostic tool in the evaluation and diagnos is of CAF. The diagnosis of coronary fistula to the main pulmonary artery can usually be made by Doppler colour flow imaging when abnormal flow signals with mosaic appearance in the pulmonary artery is visualize. It is diagnostically useful to visualize shunt flows originating from the exit of a CAF. However, sometimes fistulas to the pulmonary artery may be difficult to detect. Nowadays, a contrast echocardiography and live three-dimensional transthoracic echocardiography can be useful in demonstration of coronary fistula[4],[7].

Transoesophageal echocardiography is capable of precisely demonstrating the origin, the course and the drainage site of the fistula, but sometimes like in presented case it may fail.

Although noninvasive imaging may facilitate the 
diagnosis and identification of the orig in and insertion of CAF, coronary angiography and MDCT are necessary for the precise delineation of coronary anatomy, for assessment of hemodynamics, and to show the presence of concomitant atherosclerosis and other structural anomalies.

Ergo metry or stress/rest myocardial perfusion imaging are required to determine the possible ischemic changes in the heart. In most cases bicycle/treadmill ergometry and stress/rest Tc-99m Myoview SPECT may be useful for coronary flow assessment and fistula-related steal phenomenon. No evidence of the stress induced myocardial ischemia was found in presented case.

Risk stratification in CAF mostly depends on the significance of left-to-right shunt $(\mathrm{QP} / \mathrm{Qs}$ ratio $>1.5)$ and presence of the stress related reversible myocardial ischemia. In patients with non-significant CAF, conservative follow-up is strongly suggested and intervention procedures may be unnecessary. Elective closure of coronary artery fistulas by percutaneous transcatheter techniques or surgery is generally accepted in the presence of symptoms, but controversies exist in the management of asympto matic patients [8]. Based on the experience, there is no evidence that clinically silent CAF diagnosed incidentally by colour Doppler echocardiography are associated with adverse clinical outcome. Conservative management with continued follow-up of these patients appears to be appropriate. Potentially serious and hemodynamically significant CAF require accurate recognition, and at times, percutaneous or surgical correction.

\section{Conclusions}

Several imaging techniques are needed for an accurate diagnos is of coronary fistula and for the suggestion of proper further management. The patient presented in this case experienced a syncope at the time of diagnosis which was concluded as being of vasovagal etiology but its nature remains discussible. Consequently no intervention was recommended. The patient is seen in follow-up and is doing fine, participating in sports reporting no complaints, but he might require transcatheter closure or surgical treatment of fistula in case when he becomes symptomatic.

\section{ACKNOWLEDGEMENTS}

Authors wish to acknowledge Claudia Gibarti, MD for performing MDCT scanning, Juraj Cencarik, MD and Jozef Stasko, MD for provid ing coronary angiography. Written consent was obtained from the patient for publication of this case report.

\section{REFERENCES}

[1] Manqukia CV Coronary artery fistula. Ann Thorac Surg. 2012; 93(6):2084-92.

[2] Said SA. Current characteristics of congenital coronary artery fistulas in adults: A decade of global experience. World $\mathrm{J}$ Cardiol. 2011; 3(8):267-77.

[3] Dimitrakakis G, Challoumas D, Moschonas K, Dimitrakaki IA. eComment. Surgical management of coronary-to-pulmonary artery fistulas. Interact Cardiovasc Thorac Surg. 2012 Jan;14(1):104.

[4] Mehta D, Nanda NC, Vengala S et al. Live three-dimensional transthoracic echocardiographic demonstration of coronary artery topulmonary artery fistula. Am J Geriatr Cardiol. 2005; 14(1):42-4.

[5] Rubini G, Ettorre GC, Seb astiani M, Bovenzi F. Evaluation of hemodynamic significance of arteriovenous coronary fistulas: diagnostic integration of coronary angiography and stress/rest myocardial scintigraphy. Radiol Med. 2000; 100(6):453-8.

[6] Gowda RM, Vasavada BC, Khan IA. Coronary artery fistulas: clinical and therapeutic considerations. Int J Cardiol. 2006; 107(1):7-10.

[7] Hong SM, Yoon SJ, Rim SJ. Contrast echo - a simple diagnostic tool for a coronary fistula. Korean Circ J. 2012 Mar; 42(3):205-7.

[8] Canga Y, Ozcan KS, Emre A et al. Coronary artery fistula: Review of 54 case from single center experience. Cardiol J. 2012; 19(3):278-86. 